\section{Vacinação contra a hepatite $B$ entre trabalhadores da atenção básica à saúde}

\author{
Hepatitis B vaccination among primary health care \\ workers
}

\footnotetext{
1 Departamento de Saúde Pública, Universidade

Federal de Santa Catarina, Florianópolis, Brasil.

2 Departamento de Medicina Social, Universidade Federal de Pelotas, Pelotas, Brasil.

Correspondência L. P. Garcia Departamento de Saúde Pública, Centro de Ciências da Saúde, Universidade Federal de Santa Catarina. Campus Universitário Trindade, Florianópolis, SC 88040-900, Brasil. leilapg@matrix.com.br
}

\section{Abstract}

Health care workers' immunization against hepatitis $B$ is an essential measure to avoid occupational transmission of hepatitis $B$ virus at primary health care centers. The aims of this study were to investigate the prevalence of complete-series vaccination against hepatitis $B$, estimate the prevalence of confirmed immunity, and verify the factors associated with complete-series vaccination among primary health care workers in Florianópolis, Santa Catarina State, Southern Brazil. A total of 1,249 primary health care workers participated in this study. The prevalence of complete hepatitis B vaccination was $64.61 \%$, and $29.82 \%$ of workers indicated knowing they were immunized after taking a serological test to confirm immunity. In the adjusted analysis, complete-series vaccination was positively associated with higher level of schooling and contact with potentially infectious materials or sharps, and negatively associated with precarious employment status and current smoking. Educational measures are recommended to achieve vaccination of health workers who have not been vaccinated or have not completed the series and to inform on the need for vaccine response monitoring.

Health Manpower; Occupational Health; Primary Health Care; Hepatitis B Vaccines; Hepatitis B
Leila Posenato Garcia 1

Luiz Augusto Facchini 2

\section{Introdução}

A hepatite B apresenta distribuição universal. Estima-se que aproximadamente dois bilhões de pessoas, um terço da população mundial, já tiveram contato com o vírus da hepatite $\mathrm{B}(\mathrm{HBV}) \mathrm{e}$ 325 milhões se tornaram portadores crônicos. As infecções pelo HBV representam a décima causa de morte em todo o mundo e resultam em 500 mil a 1,2 milhão de mortes por ano, causadas por hepatite crônica, cirrose e carcinoma hepatocelular 1 .

No Brasil, a doença apresenta endemicidade variável. Endemicidades baixas (até $2 \%$ ) e intermediárias (2\% a $7 \%$ ) são predominantes, contudo algumas regiões como o sudeste do Pará, oeste de Santa Catarina e oeste do Paraná apresentam alta endemicidade (superior a 7\%) 2 . Considerando que muitos indivíduos infectados são assintomáticos e que as infecções sintomáticas são insuficientemente notificadas, espera-se que a freqüência da hepatite B seja subestimada. O Ministério da Saúde estima que, no Brasil, pelo menos $15 \%$ da população já esteviveram em contato com o HBV e que $1 \%$ apresenta doença crônica relacionada a este vírus ${ }^{3}$.

A hepatite B é a doença ocupacional infecciosa mais importante para os trabalhadores da saúde 4 . Exposições percutâneas ou de mucosas ao sangue de indivíduos infectados pelo HBV representam a principal fonte de transmissão ocupacional, já que quantidades diminutas de sangue 
são suficientes para transmitir a infecção. O HBV também está presente em outros fluidos corporais, incluindo a saliva, que também podem ser transmissores. A transmissão por meio de respingo de sangue à mucosa ocular e mordedura já foi documentada ${ }^{5}$. Além disso, a elevada resistência ambiental do HBV - que sobrevive mais de uma semana no sangue seco em temperatura ambiente e é resistente a detergentes comuns e álcool - associada ao fato de que muitos profissionais da saúde infectados pelo HBV não recordam ter sofrido exposição a sangue contaminado, leva a crer que muitas infecções ocupacionais resultam da inoculação do HBV em lesões cutâneas (como arranhões, abrasões, queimaduras) ou em mucosas 6. O HBV é mais facilmente transmitido após acidente envolvendo fluidos corporais e apresenta maior grau de infectividade do que o vírus da hepatite $\mathrm{C}(\mathrm{HCV})$ e o vírus da imunodeficiência humana (HIV) 7 .

No Brasil, a vacinação contra a hepatite B é recomendada universalmente para recém-nascidos, adolescentes até 19 anos ${ }^{3}$ e pessoas com risco acrescido para adquirir a infecção, entre elas os trabalhadores da saúde. A vacina é administrada em três doses (0, 1 e 6 meses), sendo a realização do esquema vacinal completo necessária para a imunização. Contudo, aproximadamente $10 \%$ a $20 \%$ dos indivíduos vacinados não alcançam os títulos protetores de anticorpos 7. Para os trabalhadores da saúde, o Ministério da Saúde 3 recomenda que, 30 dias após a administração da última dose do esquema vacinal contra a hepatite $\mathrm{B}$, sejam realizados exames sorológicos para controle dos títulos de anticorpos.

A maioria dos estudos sobre hepatite B entre trabalhadores da saúde foi realizada no âmbito hospitalar. Poucos estudos foram realizados com trabalhadores da atenção básica à saúde 8,9.

O presente estudo tem como objetivos verificar a prevalência da vacinação completa contra a hepatite B, estimar a prevalência da confirmação da imunidade e investigar os fatores associados à realização do esquema vacinal completo entre trabalhadores de unidades de saúde do Município de Florianópolis, Santa Catarina, Brasil.

\section{Métodos}

Usando-se inquérito epidemiológico, objetivou-se estudar a totalidade dos trabalhadores da atenção básica das unidades de saúde da Secretaria Municipal de Saúde de Florianópolis. O delineamento transversal foi selecionado por possibilitar a realização da coleta de dados em um período curto de tempo e com custo relativamente baixo 10 . Foram identificadas 49 unidades de saúde no município, divididas em cinco regionais de saúde (Centro, Leste, Norte, Sul e Continente) nas quais, no momento do estudo, havia 1.332 trabalhadores.

Foram entrevistados todos os trabalhadores, independentemente da ocupação, incluindo auxiliares administrativos e recepcionistas, auxiliares de enfermagem, técnicos de enfermagem, enfermeiros, médicos, serviços gerais, agentes comunitários de saúde, dentistas, auxiliares de consultório dentário, vigias, motoristas e outros trabalhadores de nível superior (assistentes sociais, psicólogos, fonoaudiólogos, farmacêuticos e nutricionistas), tratando-se, portanto, de um estudo censitário e não amostral.

Os dados foram coletados entre outubro e dezembro de 2006, por meio de questionários padronizados previamente testados com trabalhadores de uma unidade de saúde do Governo do Estado de Santa Catarina. Os questionários foram aplicados por entrevistadores treinados. O controle de qualidade, que não teve a intenção de verificar a repetibilidade, mas sim garantir que os dados foram coletados de maneira adequada pelos entrevistadores, foi realizado através de visitas da supervisora do estudo (L.P.G.) às unidades de saúde para confirmar as entrevistas realizadas.

As variáveis estudadas foram auto-referidas. As perguntas relacionadas à variável dependente foram: " $O(A) \operatorname{Sr}$ (a) tomou a vacina contra a hepatite B?". Em caso afirmativo, "quantas doses?" e "O(A) Sr(a) realizou exame de sangue para verificar se formou anticorpos contra a hepatite B?" $\mathrm{e}$, em caso afirmativo, " $O(A) \operatorname{Sr}($ a) ficou imunizado contra a hepatite B?”. Considerou-se vacinação completa contra a hepatite $\mathrm{B}$ a referência a pelo menos três doses da vacina.

Como variáveis independentes foram estudadas variáveis sócio-demográficas, ocupacionais e relacionadas à situação de saúde, apresentadas na Figura 1.

A entrada dos dados foi realizada usando-se o programa Epidata (Epidata Association, Odense, Dinamarca), com digitação dupla e posterior validação, para a correção dos possíveis erros de digitação.

As análises estatísticas foram realizadas com o programa Stata versão 9.0 (Stata Corp., College Station, Estados Unidos). Após a descrição das variáveis, foi realizada análise bivariável para estimar diferenças entre proporções, utilizando-se o teste exato de Fisher para variáveis de exposição categóricas nominais e o teste de tendência linear quando a variável de exposição era ordinal. Na análise multivariável, utilizou-se regressão de Poisson para verificar a associação entre a realização do esquema vacinal completo 


\begin{tabular}{l} 
Variáveis sócio-demográficas: \\
- sexo \\
- idade \\
- situação conjugal \\
- escolaridade \\
\\
Variáveis ocupacionais: \\
- contato com material biológico ou perfurocortantes \\
- regime de trabalho \\
- tempo de trabalho na prefeitura \\
- participação em cursos de capacitação relacionados à saúde do trabalhador \\
\hline Variáveis relacionadas à situação de saúde: \\
- tabagismo \\
- auto-avaliação de saúde \\
\hline Vacinação contra a hepatite B
\end{tabular}

e variáveis sócio-demográficas, ocupacionais e relacionadas à situação de saúde. A modelagem foi orientada pelo modelo hierárquico de análise ilustrado na Figura 1, com regressão para trás, nível por nível. Foram mantidas no modelo as variáveis que apresentaram associação estatística com o desfecho com um valor $\mathrm{p} \leq 0,20$, visando ao controle de possíveis fatores de confusão. Foram calculados intervalos de confiança de 95\% e consideradas estatisticamente significativas as associações com um valor $\mathrm{p} \leq 0,05$.

Os questionários não identificaram os trabalhadores que forneceram as informações, o que possibilitou a manutenção do anonimato dos participantes. As entrevistas foram realizadas após a assinatura do termo de consentimento livre e esclarecido pelos pesquisados. O estudo foi realizado de acordo com os princípios éticos que constam na Resolução $n^{o}$. 196/96 do Conselho Nacional de Saúde, tendo recebido aprovação prévia do Comitê de Ética em Pesquisa da Faculdade de Medicina da Universidade Federal de Pelotas.

\section{Resultados}

O número total de trabalhadores entrevistados foi de 1.249 , sendo detectadas $83(6,23 \%)$ perdas e recusas. A Figura 2 apresenta o fluxograma dos desfechos estudados. Enquanto $955(79,19 \%)$ tra- balhadores referiram ter tomado a vacina contra a hepatite B, 734 relataram ter tomado pelo menos três doses da vacina, indicando uma prevalência da vacinação completa de $64,61 \%$. A realização de exame sorológico para confirmação da imunidade foi mencionada por 373 (32,98\%) trabalhadores e $331(29,82 \%)$ indicaram saber que estavam imunizados.

A Tabela 1 apresenta o número de observações e freqüências nas categorias das variáveis estudadas. A idade média dos trabalhadores foi 37,42 anos (16-73) e a mediana 37 anos. A maioria era mulheres $(83,59 \%)$. Quase metade dos trabalhadores relatou realizar tarefas em que exista contato com material biológico ou perfurocortantes $(42,59 \%)$. O tempo de trabalho médio na prefeitura foi de 72,08 meses (0-372) e a mediana 48 meses. Os regimes de trabalho predominantes foram considerados precários (terceirizado, estágio ou outros) (49,56\%). Apenas 20,9\% dos trabalhadores relataram ter participado de cursos relacionados à saúde do trabalhador promovidos pela Secretaria de Saúde. A maioria dos trabalhadores nunca fumou $(67,25 \%)$. Quanto à auto-avaliação de saúde, $78,14 \%$ dos trabalhadores fizeram uma avaliação positiva (excelente, muito boa ou boa).

Na Figura 3 são mostradas as prevalências da vacinação completa contra a hepatite $B$, conforme a ocupação. A maior prevalência foi encontrada entre os enfermeiros $(85,19 \%)$, seguida 


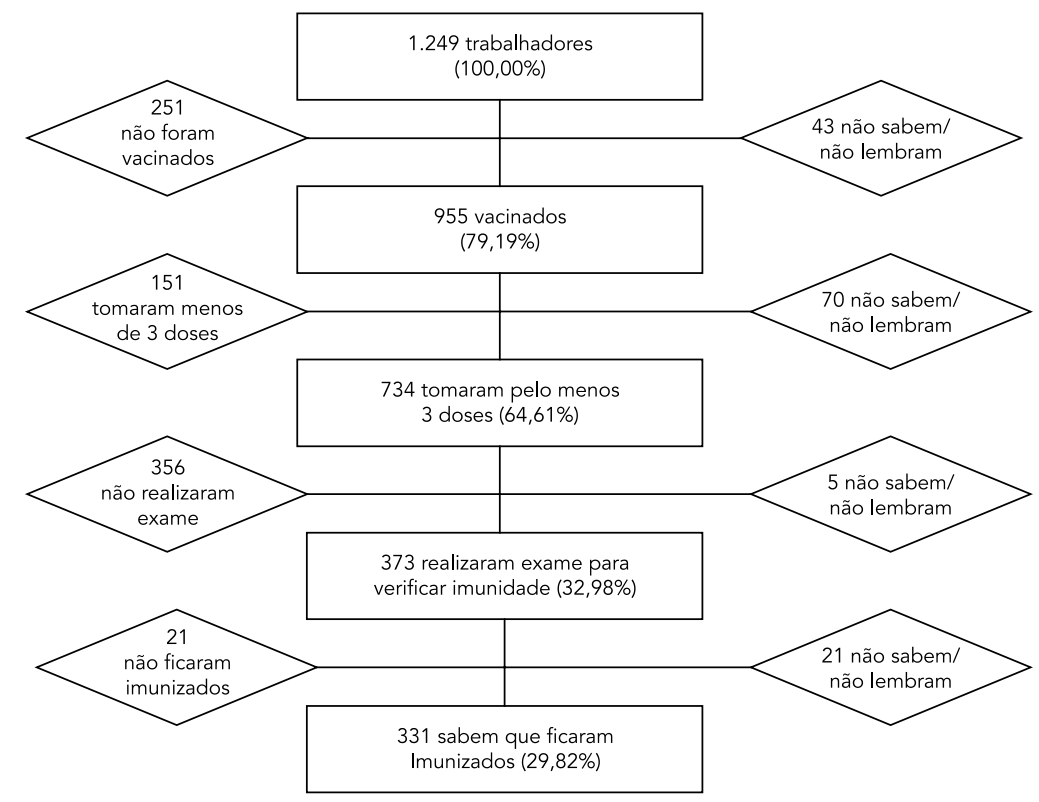

por dentistas (84,48\%) e técnicos de enfermagem $(83,09 \%)$. A menor prevalência foi encontrada entre os vigias e motoristas (38,89\%).

A Tabela 2 apresenta as análises bruta e ajustada da associação entre vacinação completa contra a hepatite B e variáveis sócio-demográficas, ocupacionais e relacionadas à situação de saúde. Na análise bruta, as variáveis idade ( $p=0,035)$, escolaridade $(p<0,001)$, contato com material biológico ou perfurocortantes ( $\mathrm{p}<0,001)$, regime de trabalho $(\mathrm{p}<0,001)$, participação em cursos de capacitação relacionados à saúde do trabalhador $(\mathrm{p}=0,011)$ e fumo atual $(\mathrm{p}<0,001)$ estavam significativamente associadas à vacinação completa contra a hepatite B. Após a análise ajustada, escolaridade $(\mathrm{p}<0,001)$, contato com material biológico ou perfurocortantes $(\mathrm{p}=0,046)$, regime de trabalho $(\mathrm{p}=0,048)$ e fumo atual $(p=0,027)$ permaneceram associadas com o desfecho, enquanto as variáveis idade ( $p=0,170$ ) e participação em cursos de capacitação relacionados à saúde do trabalhador ( $p=0,394)$ deixaram de apresentar associação estatisticamente significativa.

$\mathrm{Na}$ análise ajustada, os trabalhadores com ensino médio completo ou superior incompleto e os trabalhadores com ensino superior comple- to ou pós-graduação apresentaram chances $18 \%$ e $50 \%$ maiores, respectivamente, de ter realizado o esquema vacinal completo contra a hepatite B em relação aos trabalhadores com escolaridade até o ensino médio completo. Os trabalhadores que indicaram realizar tarefas em que pudesse haver contato com material biológico ou perfurocortantes tiveram uma chance $49 \%$ maior de ter completado o esquema vacinal, em comparação com os trabalhadores que indicaram não realizar este tipo de tarefa. Os trabalhadores sob regimes de trabalho mais precários (terceirizado, estágio, outros) apresentaram uma chance $34 \%$ menor de ter vacinação completa do que os trabalhadores sob regime estatutário. Os fumantes atuais apresentaram uma chance $19 \%$ menor de ter vacinação completa do que os não-fumantes.

\section{Discussão}

A prevalência da vacinação completa contra a hepatite B no presente estudo foi de $64,61 \%$. Essa prevalência foi superior àquela observada por Mayo-Ferrero et al. 8 que, em um estudo transversal, avaliaram todos os trabalhadores de saúde, realizado na Zona Sanitária de Santiago 
Número de observações e freqüências nas categorias das variáveis estudadas entre trabalhadores de unidades de saúde ( $n=1.249$ ). Florianópolis, Santa Catarina, Brasil, 2006.

\begin{tabular}{|c|c|c|}
\hline Variáveis & $n$ * & $\%$ \\
\hline \multicolumn{3}{|l|}{ Sexo } \\
\hline Feminino & 1.044 & 83,59 \\
\hline Masculino & 205 & 16,41 \\
\hline \multicolumn{3}{|l|}{ Idade (anos) } \\
\hline$<38$ & 633 & 50,72 \\
\hline$\geq 38$ & 615 & 49,28 \\
\hline \multicolumn{3}{|l|}{ Situação conjugal } \\
\hline Sem companheiro & 464 & 37,18 \\
\hline Com companheiro & 784 & 62,82 \\
\hline \multicolumn{3}{|l|}{ Escolaridade } \\
\hline Até ensino médio incompleto & 286 & 22,90 \\
\hline Ensino médio completo ou superior incompleto & 651 & 52,12 \\
\hline Superior completo ou pós-graduação & 312 & 24,98 \\
\hline \multicolumn{3}{|l|}{ Contato com material biológico ou perfurocortantes } \\
\hline Não & 717 & 57,41 \\
\hline Sim & 532 & 42,59 \\
\hline \multicolumn{3}{|l|}{ Regime de trabalho } \\
\hline Estatutário & 445 & 35,63 \\
\hline Contrato temporário & 185 & 14,81 \\
\hline Terceirizado, estágio, outros & 619 & 49,56 \\
\hline \multicolumn{3}{|l|}{ Tempo de trabalho na prefeitura (meses) } \\
\hline$\leq 48$ & 655 & 52,44 \\
\hline$>48$ & 594 & 47,56 \\
\hline \multicolumn{3}{|c|}{ Participação em cursos de capacitação relacionados à saúde do trabalhador } \\
\hline Não & 988 & 79,10 \\
\hline $\operatorname{Sim}$ & 261 & 20,90 \\
\hline \multicolumn{3}{|l|}{ Fumo } \\
\hline Nunca fumou & 840 & 67,25 \\
\hline Ex-fumante & 209 & 16,73 \\
\hline Fumante atual & 200 & 16,01 \\
\hline \multicolumn{3}{|l|}{ Auto-avaliação de saúde } \\
\hline Positiva & 976 & 78,14 \\
\hline Negativa & 273 & 21,86 \\
\hline
\end{tabular}

* Número de observações válidas.

de Compostela, Espanha. Foram obtidas respostas de 312 (65,6\%) deles. Quase metade (47,1\%) havia recebido pelo menos três doses da vacina contra a hepatite B. A prevalência da vacinação completa foi mais elevada entre médicos pediatras $(72,7 \%)$, seguidos pelos dentistas $(56 \%)$. No presente estudo, a maior prevalência foi observada entre enfermeiros $(85,19 \%)$, igualmente seguidos pelos dentistas (84,48\%). Em um estudo com 144 trabalhadores extra-hospitalares que haviam sofrido lesões percutâneas e exposição ocupacional a sangue em Rotterdam, Holanda 11, $65 \%$ deles estavam vacinados contra a hepatite
B, sendo a maior prevalência entre enfermeiros (81\%), seguidos por outros trabalhadores (79\%) e auxiliares de enfermagem (53\%).

Poucos estudos sobre a vacinação contra a hepatite B foram realizados com trabalhadores da atenção básica à saúde. A maioria dos estudos sobre hepatite B entre trabalhadores da saúde foi realizada no âmbito hospitalar 8,9.

A cobertura da vacinação contra a hepatite B é bastante variável em estudos realizados em diferentes países. Em um estudo transversal com 369 trabalhadores de um hospital universitário da Suécia, apenas $40 \%$ haviam completado o es- 
Prevalência da vacinação completa contra a hepatite B, conforme a ocupação, entre trabalhadores de unidades de saúde $(n=1.249)$. Florianópolis, Santa Catarina, Brasil, 2006.

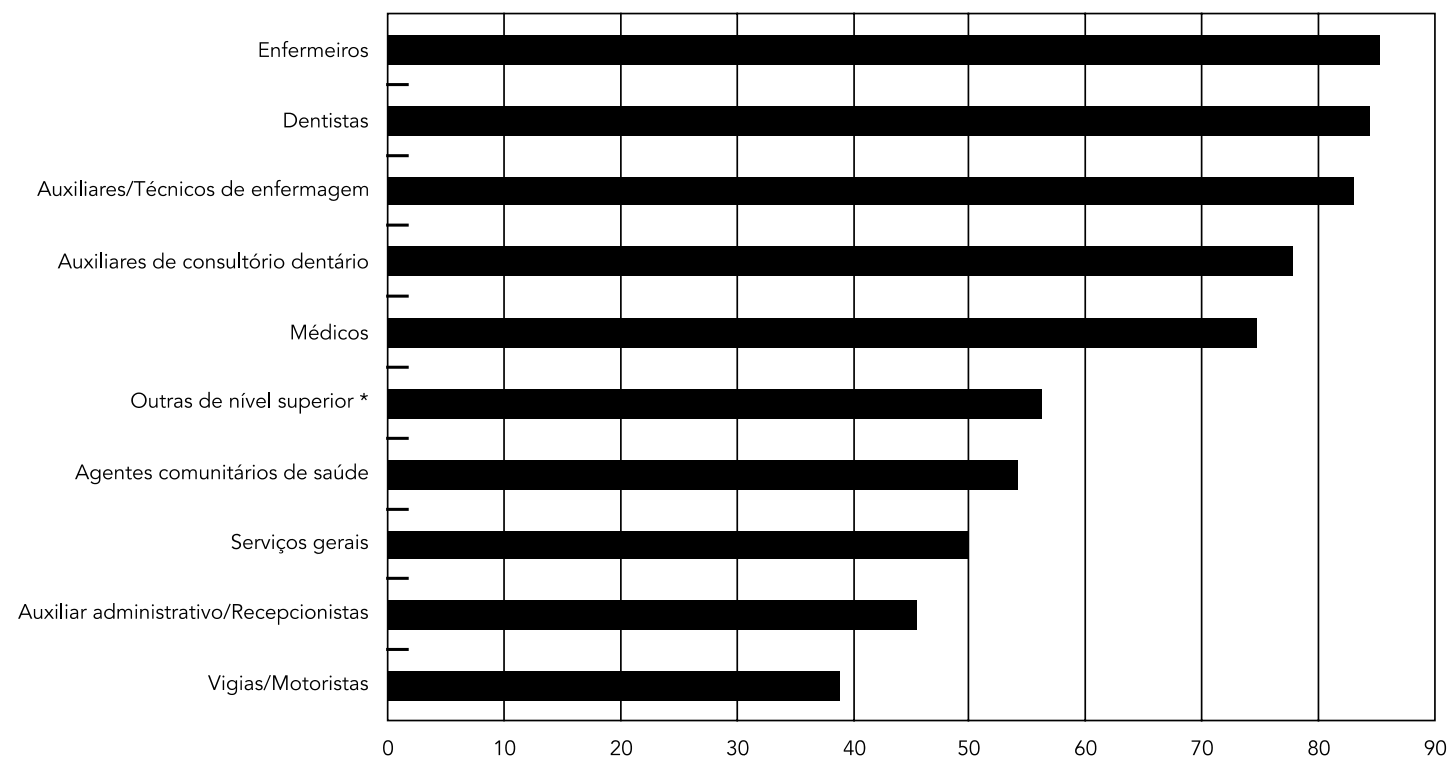

* Assistentes sociais, psicólogos, fonoaudiólogos, farmacêuticos e nutricionistas.

quema vacinal ${ }^{12}$. Um levantamento realizado na Itália em 1996 com mais de 3 mil trabalhadores de hospitais públicos demonstrou uma cobertura da vacinação média de $65 \% 13$. A cobertura da vacinação contra a hepatite B entre trabalhadores da saúde ainda está aquém da ideal na maioria dos países industrializados ${ }^{4}$. No Brasil, um estudo transversal com 298 trabalhadores de um hospital no Rio de Janeiro, verificou uma prevalência da vacinação completa contra a hepatite B de $56 \% 14$.

Além da realização do esquema vacinal completo, também é importante que os trabalhadores da saúde saibam se estão imunizados contra a hepatite B, pois, em caso negativo, eles devem ser alertados de que são suscetíveis ao HBV e devem receber imunoglobulina contra a hepatite B caso sofram exposição ao HBV. Além disso, com o passar do tempo, os títulos de anticorpos no sangue declinam, contudo os respondedores à vacina que perdem o anti-HBs detectável permanecem protegidos contra a infecção pelo HBV 5. Isso reforça a importância da realização da verificação da imunidade um mês após a administração da última dose do esquema vacinal, o que pode evitar a realização desnecessária da revacinação.
No presente estudo, 373 (32,98\%) trabalhadores realizaram exame para verificar a imunidade e 21 (5,63\% deles) detectaram que não haviam ficado imunizados. Os profissionais que não desenvolvem um nível de anticorpos adequado (anti-HBs > 10mUI/mL) após o esquema vacinal primário devem completar uma segunda série de três doses da vacina ou serem avaliados para determinar se são portadores do HBV. Quando não há resposta adequada, após o primeiro esquema vacinal, grande parte dos indivíduos responderá à repetição do esquema 4 .

Após a análise ajustada, maior escolaridade e presença de contato com material biológico ou perfurocortantes estiveram associadas com maior prevalência da vacinação completa contra a hepatite B. Já regimes de trabalho precários e fumo atual estiveram associados com menor prevalência da vacinação completa contra a hepatite B no presente estudo. A observação de que, na análise ajustada, escolaridade, contato com material biológico ou perfurocortantes, regime de trabalho e fumo atual permaneceram associados com o desfecho, enquanto idade e participação em cursos de capacitação relacionados à saúde do trabalhador deixaram de apresentar associação estatisticamente significativa pode 
Análises bruta e ajustada, através de regressão de Poisson, da associação entre vacinação completa contra a hepatite B e variáveis sócio-demográficas, ocupacionais e relacionadas à situação de saúde entre trabalhadores de unidades de saúde ( $n=1.249)$. Florianópolis, Santa Catarina, Brasil, 2006.

\begin{tabular}{|c|c|c|c|c|c|}
\hline \multirow[t]{2}{*}{ Variáveis } & \multirow{2}{*}{$\begin{array}{l}\text { Prevalência da } \\
\text { vacinação (\%) }\end{array}$} & \multicolumn{2}{|c|}{ Análise bruta } & \multicolumn{2}{|c|}{ Análise ajustada * } \\
\hline & & RP (IC95\%) & Valor $\mathrm{p}$ & RP (IC95\%) & Valor $p$ ** \\
\hline Sexo & & & 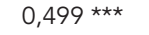 & & 0,609 \\
\hline Feminino & 67,03 & 1,00 & & 1,00 & \\
\hline Masculino & 64,15 & $0,96(0,86 ; 1,07)$ & & $1,05(0,86 ; 1,29)$ & \\
\hline Idade (anos) & & & $0,035 * \star \star$ & & 0,170 \\
\hline$<38$ & 67,67 & 1,00 & & 1,00 & \\
\hline$\geq 38$ & 61,51 & $0,91(0,83 ; 0,99)$ & & $0,90(0,78 ; 1,04)$ & \\
\hline Situação conjugal & & & $0,054 * \star \star$ & & 0,273 \\
\hline Sem companheiro & 60,99 & 1,00 & & 1,00 & \\
\hline Com companheiro & 66,71 & $1,09(0,99 ; 1,19)$ & & $1,09(0,93 ; 1,27)$ & \\
\hline Escolaridade & & & $<0,001 \#$ & & $<0,001$ \\
\hline Até ensino médio incompleto & 52,14 & 1,00 & & 1,00 & \\
\hline Ensino médio completo ou superior incompleto & 62,37 & $1,19(1,04 ; 1,37)$ & & $1,18(0,96 ; 1,45)$ & \\
\hline Superior completo ou pós-graduação & 78,62 & $1,51(1,32 ; 1,73)$ & & $1,50(1,21 ; 1,87)$ & \\
\hline Contato com material biológico ou perfurocortantes & & & $<0,001 \star \star \star \star ~$ & & 0,046 \\
\hline Não & 52,96 & 1,00 & & 1,00 & \\
\hline Sim & 78,86 & $1,49(1,36 ; 1,62)$ & & $1,22(1,00 ; 1,49)$ & \\
\hline Regime de trabalho & & & $<0,001 * \star \star$ & & 0,048 \\
\hline Estatutário & 76,47 & 1,00 & & 1,00 & \\
\hline Contrato temporário & 79,55 & $1,04(0,95 ; 1,14)$ & & $1,07(0,86 ; 1,33)$ & \\
\hline Terceirizado, estágio, outros & 50,28 & $0,66(0,59 ; 0,73)$ & & $0,79(0,62 ; 0,99)$ & \\
\hline Tempo de trabalho na prefeitura (meses) & & & $0,054 * \star \star$ & & 0,117 \\
\hline$\leq 48$ & 61,88 & 1,00 & & 1,00 & \\
\hline$>48$ & 67,51 & $1,09(1,00 ; 1,18)$ & & $1,15(0,97 ; 1,36)$ & \\
\hline \multicolumn{6}{|l|}{ Participação em cursos de capacitação relacionados } \\
\hline à saúde do trabalhador & & & $0,011 * \star \star$ & & 0,394 \\
\hline Não & 62,68 & 1,00 & & 1,00 & \\
\hline Sim & 71,49 & $1,14(1,04 ; 1,25)$ & & $1,08(0,90 ; 1,29)$ & \\
\hline Fumo atual & & & $<0,001 * \star \star$ & & 0,027 \\
\hline Não & 68,85 & 1,00 & & 1,00 & \\
\hline Sim & 55,91 & $0,81(0,73 ; 0,89)$ & & $0,83(0,71 ; 0,98)$ & \\
\hline Auto-avaliação de saúde & & & $1,000 * \star \star$ & & 0,778 \\
\hline Positiva & 64,61 & 1,00 & & 1,00 & \\
\hline Negativa & 64,63 & $1,00(0,90 ; 1,11)$ & & $1,03(0,86 ; 1,23)$ & \\
\hline
\end{tabular}

* Ajustada para idade, escolaridade, contato com material biológico ou perfurocortantes, regime de trabalho, tempo de trabalho na prefeitura, fumo atual;

** Teste de heterogeneidade;

*** Teste exato de Fischer bicaudal;

\# Teste de tendência linear.

indicar a existência de confundimento na associação destas últimas variáveis com o desfecho, devido ao efeito de outras variáveis de mesmo nível ou de níveis hierarquicamente superiores no modelo de análise.

Silva et al. 14 também observaram menor prevalência da vacinação entre serviços gerais e auxiliares de enfermagem, relacionada à baixa escolaridade das categorias, e maior prevalência da vacinação nas ocupações com maior grau de escolaridade. Outros estudos encontraram associação com variáveis como ocupação, idade, tempo de trabalho, conhecimento sobre os riscos da infecção pela hepatite B e contato com material biológico 15,16.

A terceirização está intrinsecamente ligada à precarização do trabalho, pois tem acarretado menores salários, diminuição dos níveis de 
proteção social do trabalho, ausência de benefícios e níveis mais altos de rotatividade, além de provocar fragmentação e desmobilização dos trabalhadores 17 . A observação de que a prevalência da vacinação contra a hepatite B foi significativamente menor entre os trabalhadores com formas de contratação mais precárias (terceirizados, estagiários e outros) vai de encontro aos achados de uma revisão da literatura que constatou que o emprego precário estava associado com uma deterioração da saúde do trabalhador em termos de acidentes, risco de doença, exposições perigosas e conhecimento sobre segurança e saúde ocupacional 18 .

Laurell \& Noriega 19 salientam a necessidade do estudo da relação trabalho-saúde para a compreensão de como o processo saúde-doença se articula e se expressa enquanto um processo social, visando a propor intervenções que promovam a saúde dos trabalhadores. Na perspectiva da saúde do trabalhador, a determinação social dos agravos à saúde deve ser estudada colocando em foco o processo produtivo e o trabalho 20 . No caso dos trabalhadores da atenção básica à saúde, responsáveis diretos pelos procedimentos de assistência à saúde da população, o processo de desgaste vivenciado compromete a qualidade do trabalho desenvolvido junto à comunidade 21 .

Alamillos-Ortega \& Failde-Martinez ${ }^{9}$ estudaram a prevalência de marcadores sorológicos do HBV entre 1.189 trabalhadores de hospital e 341 de atenção básica à saúde na Zona Sanitária de Jerez em Cádiz, Espanha. As prevalências de portadores do HBV e de marcadores que indicam contato prévio com o vírus foram similares em ambos os grupos. Frente a isso, deve-se salientar a importância de reforçar, não apenas nos hospitais, mas também na atenção básica, a implementação de todas as medidas preventivas disponíveis para a prevenção da hepatite B, com ênfase especial na recomendação da vacinação de todos os trabalhadores da saúde e posterior verificação do estado vacinal.

Dentre as diretrizes e propostas na área de recursos humanos para o Sistema Único de Saúde (SUS), constantes no relatório da 11ạ Conferência Nacional de Saúde 22 está a garantia da capacitação em biossegurança para os trabalhadores expostos a situação de risco. O relatório da 12a Conferência Nacional de Saúde 23 vai além, propondo a implementação de estratégias de educação permanente para qualificar trabalhadores de todas as categorias profissionais envolvidas com a área da saúde, em todos os níveis de formação, englobando, dentre os diversos conteúdos citados, saúde do trabalhador e biossegurança. Outra diretriz presente no mesmo relatório é a de assegurar que os trabalhadores de serviços ter- ceirizados tenham capacitação adequada e condições dignas de trabalho, garantindo segurança e higiene no trabalho.

No presente estudo, apenas $20,9 \%$ dos trabalhadores relataram ter participado de cursos de capacitação relacionados à saúde do trabalhador desde que iniciaram a trabalhar na Secretaria Municipal de Saúde de Florianópolis. Os recursos humanos têm papel central no SUS, portanto a garantia da saúde do trabalhador da saúde é fundamental para a prestação de serviços de qualidade à população.

A vacinação, juntamente com a aderência às medidas de precaução padrão, consciência e cuidado por parte dos trabalhadores, é fundamental para evitar a transmissão do HBV nas unidades de saúde, visando a proteger não apenas os próprios trabalhadores, mas também seus familiares e pacientes. Ela é a maneira mais eficaz na prevenção da infecção aguda ou crônica e também na eliminação da transmissão do vírus 5 .

A situação apontada no presente estudo, com tantos trabalhadores não tendo sido vacinados e grande parte dos vacinados não conhecendo seu estado sorológico frente à hepatite $\mathrm{B}$, é preocupante. Fica evidente a necessidade de capacitação dos trabalhadores da atenção básica quanto à biossegurança, saúde e segurança no trabalho, incluindo esclarecimentos acerca dos benefícios da vacinação contra a hepatite B e a respeito da necessidade do monitoramento da resposta vacinal. O SUS necessita de políticas que valorizem as condições de trabalho e saúde dos trabalhadores. Também há necessidade de programas de educação permanente para os trabalhadores da atenção básica à saúde direcionados à organização, à saúde e à segurança do trabalho.

A atenção básica à saúde é política prioritária do Ministério da Saúde, desde a implantação do SUS, que universalizou o acesso aos serviços e definiu a atenção básica como porta de entrada e principal estratégia para atingir a meta "Saúde para Todos no Ano 2000", estabelecida em AlmaAta no ano de 1978 24. Devido ao reconhecimento de que o trabalho na atenção básica à saúde é essencial e determinante da efetividade das políticas e ações de saúde, os trabalhadores da saúde e o processo de trabalho na atenção básica têm sido objeto de estudos recentes 25,26. Contudo, estudos sobre a saúde dos trabalhadores da atenção básica à saúde ainda são escassos no Brasil, fato este que reforça a relevância do presente estudo.

Como limitações, se deve salientar que o presente estudo pode estar sujeito a vieses de seleção, falsa resposta, recordatório e causalidade reversa. O viés de seleção pode ocorrer se os 
indivíduos que aceitarem participar do estudo forem diferentes daqueles que se recusarem à participação, em relação a variáveis associadas à exposição ou ao desfecho ${ }^{27}$. Para tentar minimizar esse possível viés foram feitas pelo menos três tentativas para reverter as perdas e recusas, que contabilizaram $6,23 \%$ da população, sendo uma proporção aceitável. Sempre que foi possível houve retorno às unidades de saúde para entrevistar os trabalhadores que se encontravam afastados ou em férias, após o seu retorno.

$\mathrm{O}$ viés de falsa resposta está relacionado a questões embaraçosas, quando os indivíduos podem responder falsamente por temor de serem repreendidos ou denunciados 27 . Tendo em vista a natureza desta investigação, pode-se especular que os resultados superestimem a prevalência da vacinação contra a hepatite $\mathrm{B}$, já que as pessoas tendem a reportar comportamentos aceitáveis mesmo quando não os adotam. Para minimizar esse viés, os entrevistadores foram treinados para informar e esclarecer os participantes, salientando que os dados se destinam exclusivamente à pesquisa, que os sujeitos não serão identificados, garantindo a confidencialidade das informações. Outra estratégia para minimizar a possibilidade de ocorrência desse tipo de viés foi a realização da entrevista em local reservado, de modo que as respostas do entrevistado fossem ouvidas apenas pelo entrevistador.

Considerando que os dados foram coletados retrospectivamente por meio de questionários, eles estão sujeitos a viés de recordatório, que poderia resultar em uma medida de prevalência da vacinação subestimada. Contudo, como a vacinação contra a hepatite B é feita com três doses, que são feitas em datas determinadas e controle no cartão de vacinação, é provável que aquelas pessoas que realmente foram vacinadas lembrem deste fato.

A prevalência da vacinação completa também pode ter sido subestimada devido a não ter sido investigado se os sujeitos vacinados com menos de três doses estavam no intervalo entre doses e viriam a completar o esquema vacinal. Por se tratar de um estudo transversal, o presente trabalho está sujeito a viés de causalidade reversa, que ocorre quando a aparente exposição é conseqüência do desfecho. Quando exposição e desfecho são coletados simultaneamente, deve-se ter muita cautela ao determinar relações de causalidade. Outros autores encontraram associação entre vacinação contra a hepatite B e ocorrência de acidentes de trabalho envolvendo material biológico 16 . Visando a evitar o viés de causalidade reversa, no presente estudo esta hipótese não foi testada, pois é procedimento padrão vacinar os trabalhadores não-imunizados após sofrerem acidente com exposição ocupacional a material biológico.

As causas da não-vacinação não foram investigadas no presente estudo. Briggs \& Thomas 28 investigaram as razões para que os trabalhadores da saúde do Distrito Sanitário de Croydon, sul de Londres, não tivessem tomado a vacina contra a hepatite B. Dentre as razões observadas estavam medo de efeitos colaterais ou injeções, concepções erradas sobre a transmissão da hepatite B, o uso alternativo de vacina homeopática, pressão do trabalho, dificuldades em obter a vacina, esquecimento e "inércia”. Martins e Barreto 29 também investigaram as razões alegadas para a nãovacinação, a razão mais citada foi a necessidade de mais informações.

Para estudos futuros recomenda-se a replicação de inquéritos epidemiológicos com o objetivo de verificar a cobertura vacinal relativa às diversas vacinas que são recomendadas para os trabalhadores da saúde em diferentes populações de trabalhadores da atenção básica. Também se recomenda a investigação das causas da nãoaderência à imunização, visando a direcionar a implementação de ações que resultem na ampliação da cobertura vacinal nessas populações. 


\section{Resumo}

A vacinação dos trabalhadores da saúde contra a hepatite B é fundamental para evitar a transmissão ocupacional do vírus nas unidades de atenção básica à saúde. Os objetivos deste inquérito epidemiológico foram verificar a prevalência da vacinação completa contra a hepatite B, estimar a prevalência da confirmação da imunidade e investigar os fatores associados à realização do esquema vacinal completo entre trabalhadores de unidades de saúde do Município de Florianópolis, Santa Catarina, Brasil. Participaram do estudo 1.249 trabalhadores. A prevalência da vacinação completa contra a hepatite B foi de $64,61 \%$ e 29,82\% dos trabalhadores indicaram saber que estavam imunizados após a realização de exame sorológico para confirmação da imunidade. Na análise ajustada, a vacinação foi positivamente associada com maior escolaridade e contato com material biológico ou materiais perfurocortantes durante o trabalho, e negativamente associada com regimes de trabalho precários e fumo atual. São recomendadas medidas educativas visando à vacinação daqueles que não o fizeram ou não completaram o esquema vacinal e informação sobre a necessidade do monitoramento da resposta vacinal.

Recursos Humanos em Saúde; Atenção Primária à Saúde; Saúde do Trabalhador, Vacinas contra Hepatite $B$; Hepatite B

\section{Referências}

1. World Health Organization. The world health report 1997. Geneva: World Health Organization; 1997.

2. Departamento de Análise de Situação de Saúde, Secretaria de Vigilância em Saúde, Ministério da Saúde. Situação da prevenção e controle das doenças transmissíveis no Brasil. In: Ministério da Saúde, organizador. Saúde Brasil 2004: uma análise da situação de saúde. Brasília: Ministério da Saúde; 2004.

3. Ministério da Saúde. Programa nacional de hepatites virais. Avaliação da assistência às hepatites virais no Brasil. Brasília: Ministério da Saúde; 2002.

4. Bonanni P, Bonaccorsi G. Vaccination against hepatitis B in health care workers. Vaccine 2001; 19:2389-94.

5. Centers for Disease Control and Prevention. Guidelines for viral hepatitis surveillance and case management. Atlanta: Centers for Disease Control and Prevention; 2005.

\section{Colaboradores}

L. P. Garcia e L. A. Facchini contribuíram na concepção do estudo. L. P. Garcia coordenou o trabalho de campo do estudo e o processamento dos dados. L. A. Facchini orientou essas etapas. L. P. Garcia e L. A. Facchini realizaram a análise dos dados, interpretação e redação final do artigo.

\section{Agradecimentos}

Os autores agradecem à Secretaria Municipal de Saúde de Florianópolis pelo apoio para a realização deste estudo. Agradecem também aos coordenadores das unidades de saúde e trabalhadores entrevistados pela colaboração. O presente estudo recebeu financiamento do Conselho Nacional de Desenvolvimento Científico e Tecnológico (CNPq) na modalidade apoio à tese de doutorado (número do processo: 402308/2005-0 - Ed 492005).
6. Williams IT, Perz JF, Bell BP. Viral hepatitis transmission in ambulatory health care settings. Clin Infect Dis 2004; 38:1592-8.

7. U.S. Public Health Service Updated U.S. Public Health Service guidelines for the management of occupational exposures to HBV, HCV and HIV and recommendations for postexposure prophylaxis. MMWR Recomm Rep 2001; 50(RR-11):1-52.

8. Mayo-Ferrero F, Smyth-Charmosa EF, Figueiras A. Vacunación frente al vírus de la hepatitis B em personal sanitario de atención primaria: prevalencia, factores de influencia y necesidades. Aten Primaria 1998; 22:13-20.

9. Alamillos-Ortega P, Failde-Martinez I. Prevalencia de marcadores serológicos del vírus de la hepatitis B en trabajadores de hospital y de atención primaria de salud en la zona de Jerez (Cádiz). Aten Primaria 1999; 23:121-217.

10. Pereira MG. Epidemiologia: teoria e prática. Rio de Janeiro: Editora Guanabara Koogan; 1995. 
11. Vos D, Götz HM, Richardus JH. Needlestick injury and accidental exposure to blood: the need for improving the hepatitis B vaccination grade among health care workers outside the hospital. Am J Infect Control 2006; 34:610-2.

12. Dannetun E, Tegnell A, Torner A, Giesecke J. Coverage of hepatitis B vaccination in Swedish health care workers. J Hosp Infect 2006; 63:201-4.

13. Stroffolini T, Petrosilo N, Ippolito G, Lopalco A, Sagliocca L, Adamo B, et al. Hepatitis B vaccination coverage among healthcare workers in Italy. Infect Control Hosp Epidemiol 1998; 19:789-91.

14. Silva RJO, Athayde MJPM, Silva LGP, Braga EA, Giordano MV, Pedrosa ML. Vacinação anti-hepatite B em profissionais da saúde. DST J Bras Doenças Sex Transm 2003; 15:51-5.

15. Israsena S, Kamolratanakul P, Sakulramrung R. Factors influencing acceptance of hepatitis $\mathrm{B}$ vaccination by hospital personnel in na área hyperendemic for hepatitis B. Am J Gastroenterol 1992; 87:1807-9.

16. Doebbeling BN, Ferguson KJ, Kohout FJ. Predictors of hepatitis B vaccine acceptance in health care workers. Med Care 1996; 34:58-72.

17. Cherchglia ML. Terceirização do trabalho nos serviços de saúde: alguns aspectos legais, conceituais e pragmáticos. In: Santana P, Castro J, organizadores. Capacitação em desenvolvimento de recursos humanos de saúde. Natal: Editora da UFRN; 1999. p. 367-85.

18. Quinlan M, Mayhew C, Bohle P. The global expansion of precarious employment, work disorganization and consequences for occupational health: a review of recent research. Int J Health Serv 2001; 31:335-414

19. Laurell AC, Noriega M. Processo de produção e saúde: trabalho e desgaste operário. São Paulo: Editora Hucitec; 1989.

20. Mendes R, Dias EC. Saúde do trabalhador. In: Rouquayrol M, organizador. Epidemiologia e saúde. 4 a Ed. Rio de Janeiro: Medsi Editora; 1993. p. 431-56.
21. Magalhães MJO, Muniz HP, Costa JD, Ribeiro FF Análise da relação entre a situação de trabalho e a saúde dos profissionais da unidade de Saúde da Família/Grotão II. In: II Mostra Nacional de Produções em Saúde da Família. Brasília: Ministério da Saúde; 2005.

22. Conselho Nacional de Saúde. Desenvolvimento de trabalhadores em saúde. In: 11ạ Conferência Nacional de Saúde. O Brasil falando como quer ser tratado. Efetivando o SUS: Acesso, qualidade e humanização na atenção à saúde com controle social. Brasília: Ministério da Saúde; 2000. p. 162-5. (Série Histórica do CNS, 2).

23. Conselho Nacional de Saúde. Eixo temático VII: o trabalho na saúde. In: 12a Conferência Nacional de Saúde. Conferência Sergio Arouca: relatório final. Brasília: Ministério da Saúde; 2000. p. 115-31.

24. World Health Organization. Primary health care - Report of the International Conference on PHC. Geneva: World Health Organization; 1978.

25. Facchini LA, Piccini RX, Tomasi E, Thumé E, Silveira DS, Siqueira FV, et al. Desempenho do PSF no Sul e no Nordeste do Brasil: avaliação institucional e epidemiológica da atenção básica à saúde. Ciênc Saúde Coletiva 2006; 11:669-81.

26. Ribeiro EM, Pires D, Blank VL. A teorização sobre processo de trabalho em saúde como instrumental para análise do trabalho no Programa Saúde da Família. Cad Saúde Pública 2004; 20:438-46.

27. Werneck GL, Almeida LM. Validade em estudos epidemiológicos. In: Medronho R, organizador. Epidemiologia. São Paulo: Editora Atheneu; 2003 p. 199-212.

28. Briggs MJ, Thomas MJ. Obstacles to hepatitis B vaccine uptake by health care workers. Public Health 1994; 108:137-48.

29. Martins AM, Barreto SM. Vacinação contra a hepatite B entre cirurgiões-dentistas. Rev Saúde Pública 2003; 37:333-8.

Recebido em 06/Mar/2007

Versão final reapresentada em 11/Set/2007

Aprovado em 25/Out/2007 